\title{
Promoting Reproductive Health Using Telemedicine: A Prospective Study among Rural Appalachian High School Teens
}

Raj Singh, MSIII ${ }^{1}$, Mohit Harsh, MSIII ${ }^{1}$, Keegan Mullins, MSIII ${ }^{1}$, Brian Dunlap, MD ${ }^{1}$, Jennie L. Yoost, $\mathrm{MD}^{1}$

\section{Author Affiliations:}

1. Marshall University Joan C. Edwards School of Medicine, Huntington, WV

The authors have no financial disclosures to declare and no conflicts of interest to report.

\section{Corresponding Author:}

Jennie L. Yoost, MD, MSc, FACOG

Department of Obstetrics \& Gynecology

Marshall University

Joan C. Edwards School of Medicine

Huntington, WV 25701

Email: yoost@marshall.edu 


\section{Abstract:}

Introduction:

Telemedicine allows rural underserved populations access to medical resources that may not be available in their communities. Following promising results with a telemedicine project aimed at educating female students in McDowell County, West Virginia on reproductive health (RH) in 2015, we conducted a follow-up study including both male and female high school students during the 2016 school year.

\section{Materials and Methods:}

Telemedicine sessions on RH were incorporated into existing afterschool programs at two rural high schools. Students' knowledge on RH was assessed via pre-test, immediate post-test, and 6month post-test evaluations. RH was taught by medical students and faculty at a distal university.

Results:

77 students participated and 37 students completed a 6-month follow-up survey. Statistically significant increases in knowledge scores regarding both birth control and sexually transmitted disease prevention were noted at 6-month follow-up. A larger proportion of students reported "always" utilizing birth control ( $35.3 \%$ vs. $64.7 \%)$ as well as condom usage ( $46.2 \%$ vs. $58.8 \%)$ at six months compared to before the intervention, but was not statistically significant. The intervention was rated as "effective" or "very effective" by $91.9 \%$ of participants, and $75.6 \%$ stated they were "very likely" to participate the future.

\section{Discussion:}

Telemedicine is a promising and sustainable tool in teaching $\mathrm{RH}$ to rural underserved areas.

Keywords: Tele-education, reproductive health, sex education, contraception, sexually transmitted disease prevention

\section{Introduction}

Teen pregnancy is a significant problem in the United States (US) compared to other similar industrialized countries. Compared to Canadian adolescents, American teens are estimated to be 2.5 times more likely to have children. ${ }^{1}$ The national teen birth rate among females age 15-19 in 2015 was 22 per 1000 , with approximately $27.3 \%$ of US high school students reporting sexual activity by $9^{\text {th }}$ grade, and $58.1 \%$ by $12^{\text {th }}$ grade..$^{2,3}$ There are significant variations at both state and county-levels in teen pregnancy rates. In West Virginia (WV), the teen birth rate was 31.9 per 1000 in 2015 with roughly $46.7 \%$ of WV high school students reporting sexual activity., 3 Notably, some areas of WV report rates nearly three times higher than that of the national average, with no significant declines over the past few years. In 2013, McDowell County (MC), located in southern WV, had a teen birth rate of 78.1 per $1000 .{ }^{4}$ Teen pregnancy has been 
hypothesized to be more common in communities where adolescents feel they have poor economic prospects due to lack of education or inadequate financial resources. ${ }^{1}$ In MC, the high school dropout rate in 2014 was $14 \%$, and $45.4 \%$ of children under 18 in 2013 were estimated to live in homes below the federal poverty level. ${ }^{4}$ This may explain the high rate of births in MC to mothers who had not completed high school (estimated to be $30.8 \%$ of all births in 2013). ${ }^{4}$ As such, resource-challenged communities like MC are faced with a vicious cycle, as low socioeconomic status is a risk-factor for early sexual debut and teen pregnancy due to perceived poor future economic prospects. ${ }^{1}$

In 2013, 60.3\% of WV high school students that were sexually active reported not using a condom during their last sexual intercourse encounter. Even among those using preventive measures, incorrect use of effective methods contributes to high contraceptive failure rates and sexually transmitted diseases (STD) in teens. ${ }^{5}$ As such, early interventions aimed at educating the adolescent population on reproductive health (RH), especially in areas that are at-risk for early initial sexual intercourse with subsequent high teen pregnancy rates, are key.

Telemedicine is the provision of medical care or education from a distal site through the use of teleconferencing communication. We have previously reported on results from a pilot study that examined whether a telemedicine educational intervention for female adolescent students in MC could successfully promote knowledge of $\mathrm{RH}^{6}{ }^{6}$ Following promising initial findings, we continued the program the following academic year and expanded the cohort to include male adolescents. Here, we report on outcomes following the second year of this program with the inclusion of males. The goals of this study were to examine the effectiveness of this intervention in disseminating knowledge on RH topics as well as examine students' perceptions of the effectiveness of telemedicine as a teaching modality.

\section{Materials and Methods}

Telemedicine sessions, entitled "Teen Talk," were incorporated into existing afterschool programing within the two high schools in McDowell County. Participation in the afterschool program was optional, and students could choose to attend the sessions or to complete any surveys. The telemedicine sessions began in March 2016 and continued through May 2016. The program consisted of one telemedicine session a week for seven weeks with separate sessions for male and female students. Each session was approximately one hour in duration and included either a reproductive health or life skill topic. Reproductive health topics included male and female anatomy, physiology of reproduction, contraception and abstinence, STD prevention, and other specific teen health concerns. The life skill sessions included diet and exercise, mental health and stress management, sleep, relationship health, self-esteem and career planning. Life skill topics were included in the program to encourage participation by promoting a variety of health topics aside from RH. A website, www.marshallteentalk.org, was developed to enable student access all materials, pre and post-tests and resources discussed during the telemedicine sessions. Students were also able to submit anonymous questions through this website.

Sessions were taught by both medical students and faculty mentors at a distal medical school. The telemedicine sessions were interactive, allowing time for questions and answers. The faculty mentors and medical students conjointly developed and reviewed materials for the sessions. 
Faculty mentors included two physicians with expertise in adolescent health. Visual aids, such as birth control models, were shipped to each school prior to and distributed during the sessions to students in order to corroborate the understanding of topics. In addition, posters and flip charts were utilized to enhance learning. A large majority of the material was expanded upon from the initial program's curriculum ${ }^{6}$ in response to student feedback.

At the start of the program, student participants completed a demographic survey with nonidentifying information such as risk factor assessments for teen pregnancy and early school dropout. Prior to each RH session, baseline reproductive knowledge on the day's topic was assessed by a short pre-test. Post-tests were administered at the end of each session, to assess knowledge and measures of self-efficacy, or how likely the students would be able to put what they learned into practice. After the final session, questions regarding the acceptability, comprehension, and ease of use of telemedicine were assessed. Six months after the telemedicine program, the participants were asked to complete a final survey assessing knowledge retention and self-efficacy.

The demographic survey, pre and post-tests, and six-month post-test assessment were submitted using an online survey tool linked to the website. Each high school had multiple iPads available for use during the telemedicine sessions for survey completion and for students to view the website material. Each student was assigned an ID number so that participation and knowledge was followed prospectively. Two McDowell County teachers within the afterschool program served as coordinators, distributing materials and iPads for survey completion, and keeping attendance. Students were offered a gift card incentive of $\$ 5$ per session for attendance and $\$ 10$ for demographic survey completion. They were offered an incentive of a $\$ 25$ gift card for sixmonth survey completion.

Immediate outcomes including change in RH knowledge were evaluated with pre and post-tests. A set of 10 knowledge based questions were included in the contraception and STD session and assessed pre-test, post-test and knowledge retention at six months. These questions were scored based on number correct and were based off of previously studied RH questions as previously described. ${ }^{6}$ Long term outcomes such as teen pregnancy rate and school dropout rate were obtained by the McDowell County school board. Demographic and pre-test, post-test, and 6 month data were compared with two-sample test of proportions for dichotomous data or student's t-test calculated via SPSS v22. Institutional review board approval was obtained prior to study initiation.

\section{Results}

Seventy-seven students attended at least one session and the average number of participants per session was 36.8 (range: 27-47). (Table 1) A total of 42 female students and 35 male students participated in the study. Fifty-one students (66.2\%) completed the initial demographic survey. (Table 2). The average age of participants was 15.5 (range: 13-19). Sexual activity was reported in $47 \%$ of participants, with $88.3 \%$ self-reporting as heterosexual, and $62.5 \%$ of sexually active participants reported never being tested for an STD. Of those completing the initial survey, $15.6 \%$ reported that their mothers had been pregnant as teenagers (age 18 years and younger), but $45 \%$ reported that their mothers had been pregnant by the age of 21 . Only 
$37.2 \%$ of subjects' mothers and $17.5 \%$ of subjects' fathers achieved education beyond high school. Four subjects (7.8\%) reported having already been pregnant, and three subjects (5.8\%) reported a desire to become pregnant within the next year.

Table 1: Telemedicine sessions offered during the after school program and number of high school students attending.

\begin{tabular}{ll}
\hline Session Title & Number of Participants \\
\hline Birth Control & 40 \\
STD Prevention & 36 \\
Self-Esteem & 24 \\
Relationship Health & 31 \\
Nutrition \& Exercise & 27 \\
Stress Management & 36 \\
Career Planning & 47 \\
\hline
\end{tabular}

Table 2: Demographic and intake survey responses among high school participants $(\mathrm{N}=51)$

\begin{tabular}{lll}
\hline Intake Data & & $\mathrm{N}(\%)$ \\
\hline Age & Mean 15.5 (range 13-19) & \\
Grade: & $9^{\text {th }}$ & $17(33.3)$ \\
& $10^{\text {th }}$ & $18(35.2)$ \\
& $11^{\text {th }}$ & $8(15.6)$ \\
Race & 12 th & $5(9.8)$ \\
& White & $29(56.8)$ \\
& Black & $19(37.2)$ \\
Living Situation & Other & $3(5.8)$ \\
& Both Parents & $20(39.2)$ \\
& Single Mom & $16(31.3)$ \\
& Single Dad & 2 \\
& Grandparents & 5 \\
& Significant Other & 2 \\
Highest level of education achieved & Other & $5(9.9)$ \\
by mother & College & $17(33.3)$ \\
Highest level of education achieved & Post-graduate & $2(3.9)$ \\
by father & College & $8(15.6)$ \\
Who are you attracted to? & Post-graduate & $1(1.9)$ \\
& Heterosexual & $45(88.3)$ \\
Have you ever had sex? & Bisexual or homosexual & $6(11.7)$ \\
& Yes & $24(47)$ \\
Have you ever been tested for an & No & $27(52.9)$ \\
STD? & Yes & $9(37.5)^{*}$ \\
Do you plan on having sex within & No & $15(62.5)$ \\
the next year? & No & $15(29.4)$ \\
& Not Sure & $21(41.1)$ \\
Have you or your partner ever been & Yes & $15(29.4)$ \\
pregnant? & Yes & $4(7.8)$ \\
Would you like to become pregnant & Ye & $47(92.2)$ \\
within the next year? & No & $3(5.8)^{* *}$ \\
\hline Among & $47(92.2)$ \\
\hline
\end{tabular}

*Among those reporting sexually active $(\mathrm{n}=24)$

**Different responders from those stating already had been pregnant. 
A total of $72.5 \%(37 / 51)$ of students responded to the 6-month survey. Likert scale compliance with condom use and contraception was assessed at intake survey and at 6 months. The number of participants "always using condoms" increased during this time (46.2\% of those sexually active at intake vs. $58.8 \%$ at 6 months; $\mathrm{p}=0.49$ ). The number of those "always using birth control" also increased (35.3\% of those sexually active at intake vs. $64.7 \%$ at 6 months; $p=$ 0.08). Report of pregnancy was assessed at six months and correlated with data obtained from the school coordinators. No pregnancies or school dropouts occurred among program participants.

Subjects were also asked about the effect of pregnancy on aspects of life, such as relationships (peers, family, partner), social life, future plans, education, and finances (Figure 1). The greatest effect on the perceived negative effect of pregnancy was with future plans and finances, with a significantly larger proportion of students reporting that pregnancy would "worsen" these aspects of their life.

Figure 1: Student responses on how pregnancy could affect different aspects of their life at intake and then at 6 months after intervention.

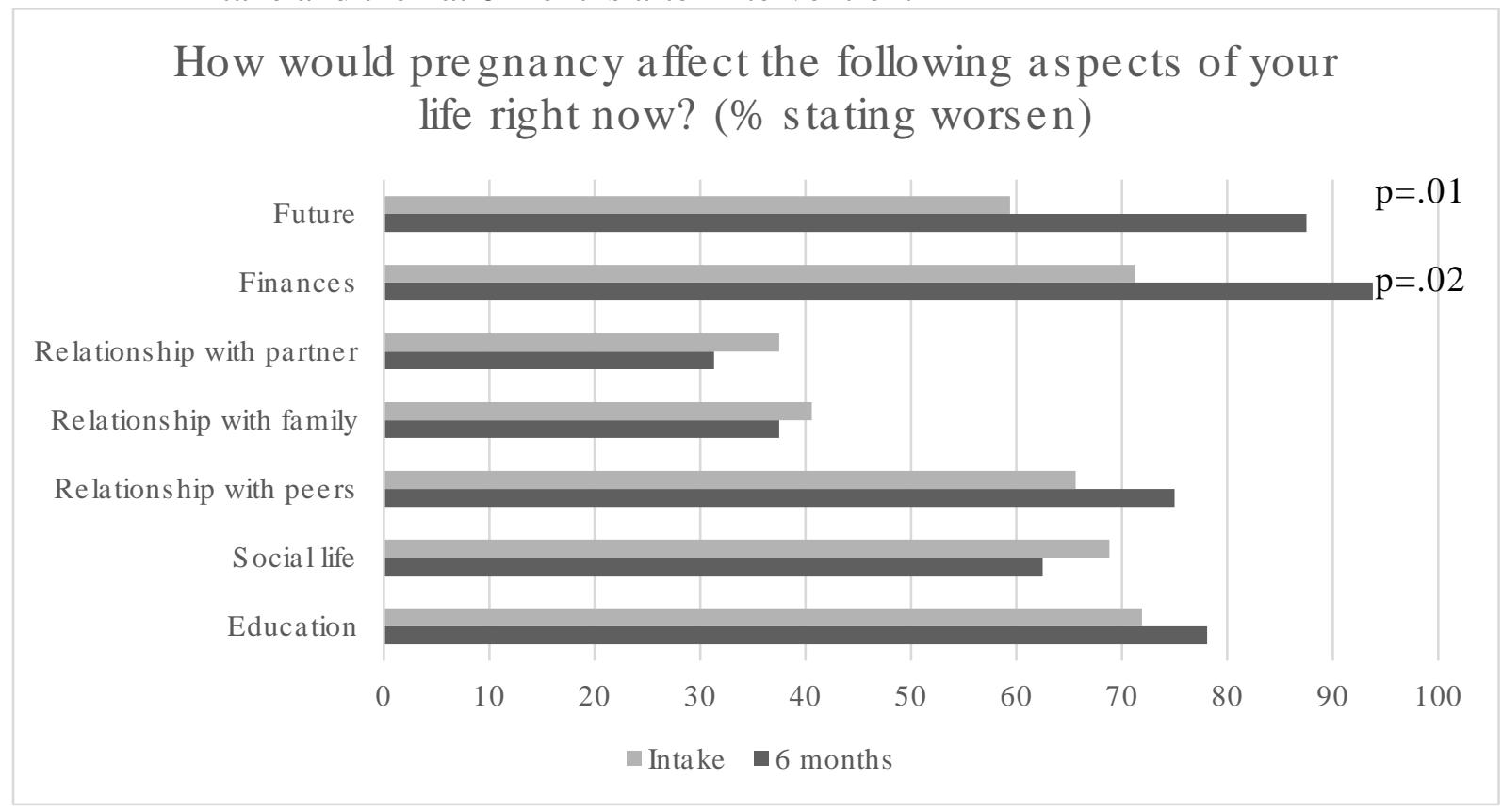

\section{Reproductive Health}

Forty students participated in the birth control session and 36 students participated in the STD prevention session. Table 3 contains results from pre and post-tests and six month assessment of RH knowledge. With respect to birth control, the mean knowledge score increased from pre-test 4.89 (SD 1.5) to post-test 9.0 (SD 1.2), $\mathrm{p}<.001$. A total of 25 students who attended the birth control session completed the 6-month survey. Knowledge score was maintained at 6 months when compared to pre-test assessment, with the mean score increasing from 4.92 (SD 1.7) to 6.64 (SD 1.95) at 6 months $(\mathrm{p}=0.002)$. However, when comparing the post-test assessment results to six month scores there was a decrease from 8.9 (SD1.4) to 6.64 (SD 1.9), $\mathrm{p}=<.001$. 
In the STD session the mean knowledge score increased from 7.0 (SD 1.8) pre-test to 8.3 (SD $1.9)$ post-test $(\mathrm{p}=0.001)$. A total of 21 students who attended the session completed the 6month survey. Knowledge score was maintained at 6 months, with mean score increasing from 7.5 (SD 1.4) to 8.6 (SD 2.0) at 6 months $(\mathrm{p}=0.05)$. When compared to post-test, the mean knowledge score did not change at 6 months (8.86 SD 1.4 vs. 8.6 SD 2.0; $\mathrm{P}=.54)$.

Table 3: Knowledge scores among participants pre-test, post-test, and at six months

\begin{tabular}{lllll}
\hline Session & Pre-Test Score & Post-Test Score & 6 Month Score & Significance \\
\hline Birth Control & 4.89 (SD 1.5) & $9.0($ SD 1.2) & $6.64(\mathrm{SD} 1.95)$ & $\mathrm{p}=<.001^{*}$ \\
& $\mathrm{~N}=40$ & $\mathrm{~N}=40$ & $\mathrm{~N}=25$ & $\mathrm{p}=.002^{* *}$ \\
& & & & $\mathrm{p}=<.001^{* * *}$ \\
STD Prevention & $7.0(\mathrm{SD} 1.8)$ & $8.3(\mathrm{SD} 1.9)$ & $8.6(\mathrm{SD} 2.0)$ & $\mathrm{p}=.001^{*}$ \\
& $\mathrm{~N}=36$ & $\mathrm{~N}=36$ & $\mathrm{~N}=21$ & $\mathrm{p}=.05^{* *}$ \\
& & & & $\mathrm{p}=.54^{* * *}$ \\
\hline
\end{tabular}

Comparison of pre-test to post-test scores*

Comparison of pre-test to six month scores**

Comparison of post-test to six month scores***

\section{Telehealth Evaluation}

At the concluding session $(\mathrm{N}=47)$ Likert scale evaluation revealed that $89.4 \%$ of subjects found the use of telehealth to be "effective" or "very effective" as a means to teach material, and $82.9 \%$ were "very confident" in the accuracy of the material provided. Majority, $91.4 \%$, of participants found the discussion to be "easy" or "very easy" to follow using the teleconferencing equipment. A total of $43.5 \%$ of participants utilized the website (www.marshallteentalk.org) for information outside of the scheduled telemedicine sessions. A total of $42 / 47(89.4 \%)$ found the material presented to be appropriate for high school teenagers. When asked how likely they would be to participate in telehealth programs in the future, $68 \%$ were very likely to attend and $68 \%$ were likely to recommend to a friend.

At the 6-month program evaluation $(\mathrm{N}=37), 35 \%$ stated that they had used the website in the past 6 months, $70.3 \%$ found the program "very effective" overall and $21.6 \%$ found it "effective." $81 \%$ were "very confident" in the material presented, and $75.6 \%$ of subjects were "very likely" to participate in a telehealth program in the future.

\section{Discussion}

This prospective study demonstrates that telemedicine can be a useful tool in teaching RH to rural teens. Reports examining the utility of telemedicine in disseminating information on RH has been very limited to date. As such, we set out to implement this educational intervention among high-risk teens, with a goal that it could be reproduced by other rural and underserved areas facing similar challenges. We previously reported on initial findings with a telemedicine RH program that included a cohort of 55 female adolescents in MC. Following this intervention, statistically significant increases in both condom use $(20 \%$ to $40 \% ; \mathrm{p}=0.04)$ and HPV vaccination ( $38 \%$ to $70 \% ; \mathrm{p}=0.001$ ) were found at 6 -month follow-up. Notably, $91.8 \%$ of the cohort participating in the study rated telehealth as a "very effective" method of providing 
information on $\mathrm{RH}^{6}{ }^{6}$ Due to positive feedback from the study participants and school board, the program expanded to include males.

This study has some limitations that merit attention. For example, the small sample size limits the power and generalizability of our findings. Though there was an increase in the proportion of students reporting utilization of condoms and birth control, none of these findings were significant likely due to the low sample size. Possible reasons for lower participation among students include timing conflicts, lack of parental encouragement, and confidentiality concerns. However, it is worth noting that the number of students attending each session remained consistent throughout the study period. Also, as this program was incorporated into an afterschool program, participation was voluntary, and no randomization or control group was possible. Notably, a few students gave feedback that it was difficult to learn from some sessions utilizing the lecture-based format.

While knowledge scores increased from pre-test to post-test and pre-test to six months for the birth control session, there was a decrease in score comparing post-test to six months. When looking at individual questions completed, more students answered the questions regarding IUD safety, use in teens, and access incorrectly at six months. This decrease may be an effect of cultural or social influences and lack of access to this method. For example, the school-based health clinics at both high schools offer contraception, but do not offer intrauterine devices.

Notable strengths of this study include the prospective design, assessment of participants' knowledge on RH immediately following the intervention and 6 months post-intervention, as well as the ability for future longitudinal follow up of subjects due to assigned ID numbers. As this study represents a continuation of an initial pilot study one-year ago, students, teachers, and parents in the community also were more likely to feel comfortable and familiar with the medical students and mentors involved.

This program is unique because it offers multiple health topics, aside from $\mathrm{RH}$. This was done to promote participation, as teens may be less willing to voluntarily attend sessions only dealing with RH. However, we found that the two RH sessions were well, if not better, attended than some of the other topics. The addition of a website to store surveys, session material, and submission of confidential questions was an improvement from the prior year. Feedback from the high school students was positive regarding the website, but suggestions for improvement included adding videos or podcasts. While iPads were used in the program, the website was also accessible on smartphones or other computers, which could allow it to be used in other settings.

The model of using medical students as teachers of the sessions is also unique. Medical students are often eager to be engaged in community outreach, have a broad knowledge base, and can serve as mentors to high school teens. The faculty involved are experts in adolescent health care and served to assure that all material taught was accurate and evidence-based. This model could be easily implemented at other university settings, and has been sustainable within our own institution. 
Telemedicine can effectively provide education on RH to adolescents in rural, resourcechallenged areas without close access to experts in such areas. This unique approach can easily be replicated in other rural counties facing similar challenges throughout WV and the US. 


\section{References}

1. Kearney MS, Levine PB. Why is the teen birth rate in the United States so high and why does it matter? The journal of economic perspectives : a journal of the American Economic Association. 2012 Spring;26(2):141-66. PubMed PMID: 22792555.

2. Martin JA, Hamilton BE, Osterman MJ. Births in the United States, 2015. NCHS data brief. 2016 Sep(258):1-8. PubMed PMID: 27648876.

3. Kann L, McManus T, Harris WA, Shanklin SL, Flint KH, Hawkins J, et al. Youth Risk Behavior Surveillance - United States, 2015. Morbidity and mortality weekly report Surveillance summaries. 2016 Jun 10;65(6):1-174. PubMed PMID: 27280474.

4. Kids Count Data Center The Annie E Casey Foundation [cited 2016 October 21, 2016]. Available from: http://datacenter.kidscount.org.

5. CDC. West Virginia 2015 State Profile [March 6, 2017]. Available from: https://www.cdc.gov/nchhstp/stateprofiles/pdf/west_virginia_profile.pdf.

6. Yoost JL, Starcher RW, King-Mallory RA, Hussain N, Hensley CA, Gress TW. The Use of Telehealth to Teach Reproductive Health to Female Rural High School Students. Journal of pediatric and adolescent gynecology. 2016 Oct 11. PubMed PMID: 27742427. 